\title{
Supersymmetry after the Higgs discovery
}

\author{
I. Antoniadis ${ }^{1, \mathrm{a}}$, D. Ghilencea ${ }^{1,2, \mathrm{~b}}$ \\ ${ }^{1}$ Department of Physics, CERN - Theory Division, 1211 Geneva 23, Switzerland \\ 2 Theoretical Physics Department, National Institute of Physics and Nuclear Engineering (IFIN-HH), 077125 Bucharest, Romania
}

(C) The Author(s) 2014. This article is published with open access at Springerlink.com

These are interesting times for theoretical and experimental high energy physics. Nearly five decades after the Higgs particle theoretical prediction (1964), the ATLAS and CMS experiments of the Large Hadron Collider (LHC) at CERN confirmed (4 July 2012) the existence of the Higgs boson of the Standard Model (SM) and, implicitly, its associated (Brout-Englert-Higgs) mechanism of electroweak (EW) symmetry breaking. This confirmation is a great triumph of theoretical high energy physics and, in particular, of the principle of symmetries that modern physics is based upon, introduced early last century by E. Noether.

Supersymmetry (SUSY) is a new symmetry that relates bosons and fermions, which has strong support at both the mathematical and the physical level. At the mathematical level, SUSY avoids the restrictions of the ColemanMandula no-go theorem by the introduction of spinorial generators (supercharges) that makes SUSY the only possibility in which space-time beyond Poincaré and internal symmetries of the S-matrix can be combined consistently (HaagLopuszanski-Sohnius). The existence of a super-Poincaré (graded Lie) algebra and its representations (superfields) further supported this new symmetry on strong mathematical grounds. Moreover, if imposed as a local symmetry, general relativity is included automatically (supergravity). It is then no surprise that SUSY is also a fundamental ingredient in string theory where it plays such a crucial role, even if no trace of this symmetry is left at low energies.

At the physical level, the motivation is even stronger, when applied to the Standard Model, to obtain a (minimal) supersymmetric extension of it that could be valid at energies as low as the $\mathrm{TeV}$ scale. The motivation is that $\mathrm{TeV}$-scale SUSY solves the mass hierarchy problem of the SM and stabilizes the EW scale in the presence of quantum corrections, by ensuring an improved ultraviolet behavior of the theory. TeVscale SUSY is consistent with a dynamical (radiative) elec-

\footnotetext{
a e-mail: ignatios.antoniadis@ @ern.ch

be-mail: dumitru.ghilencea@cern.ch
}

troweak symmetry breaking which in the Standard Model is not explained, being an ad-hoc input. Further, in SUSY models the unification of the fundamental forces in Nature (weak, strong, and electromagnetic) is naturally achieved, to realize a long-held dream of high energy physics. This unification picture is completed by the unification with gravity, as done in various string models, like the weakly coupled heterotic string. SUSY also provides an interesting dark matter candidate, consistent with thermal relic abundance calculations, which may soon be detected by accelerator- or satellite-based experiments. All these features rely to a large extent on the existence of low, TeV-scale SUSY, which is thus accessible at the ongoing LHC experiments. Exact, non-perturbative results are also possible in the presence of SUSY. The consistency of all these theoretical and phenomenological advantages of SUSY made it become the most popular candidate for "new physics" beyond the Standard Model.

One initial drawback of this theory is that it more than doubles the SM spectrum, something regarded with serious skepticism by some experimentalists and even theorists. SUSY predicts a plethora of new particles (superpartners) that so far were not detected by large and small scale physics experiments. In particular the constraints from the first run of the LHC ( 7 and $8 \mathrm{TeV}$ ) restricted significantly the parameter space of various minimal supersymmetric models, such as the constrained minimal supersymmetric standard model. Another problem is an increased fine tuning (instability) of the EW scale in some simple models, which may question the success of SUSY in solving the hierarchy problem that motivated its introduction in the first place. These problems point to the breaking mechanism of SUSY, whose details remain somewhat mysterious.

These are, however, early days in the great effort to detect SUSY experimentally. Until Run 2 of the LHC (13 and 14 $\mathrm{TeV}$ ) is performed and completed it is difficult to make definite statements about the existence of TeV-scale SUSY, even in minimal models. So far, the existence of a Higgs boson, in a (perturbative) region perfectly well compatible with SUSY, 
gives us hope that this scalar particle is only one of many other scalars that we so far failed to discover. Why should there be only a single scalar particle, but so many fermions and gauge bosons? The optimist would even say that we already have a scalar particle and with a mass range, both predicted by SUSY, the Higgs boson, so we must be on the right track.

The current volume intends to be a review of these ideas, following the development of SUSY from its very early days up to present. The order of the contributions should provide the reader with the historical development as well as the latest theoretical updates and experimental constraints from particle accelerators and dark matter searches. It is a great pleasure to bring together in this volume contributions from people who initiated or contributed significantly to the development of this theory over so many years. For a balanced point of view, the volume also includes a (last) contribution that attempts to describe the physics beyond the Standard Model in the absence of SUSY.

Beloved by many theorists or shunned by as many experimentalists, the idea of SUSY remains attractive. We are fortunate that the LHC has good chances to clarify the question if SUSY really exists near the TeV scale. Its experimental confirmation would certainly dominate particle physics for many decades to come with an impact that is hard to imagine at this moment. The alternative is that this scale is pushed higher and higher, moving this beautiful idea further away from our experimental reach. This would make theorists wonder whether they pinned their hopes for too long on a single, most beautiful but elusive idea and whether the time is ripe to re-consider our view on physics near the $\mathrm{TeV}$ scale.

Geneva, March 2014.

\section{Contents of this volume:}

1. P. Ramond, "SUSY: the early years (1966-1976)". doi:10.1140/epjc/s10052-013-2698-X

2. P. Fayet, "The supersymmetric standard model”. doi: 10.1140/epjc/s10052-014-2837-z

3. I. Melzer-Pellmann (CMS), P. Pralavorio (ATLAS), "Lessons for SUSY from the LHC after the first run". doi:10.1140/epjc/s10052-014-2801-y

4. J. Ellis, "Supersymmetric fits after the Higgs discovery and implications for model building”. doi:10.1140/epjc/ s10052-014-2732-7

5. A. Djouadi, "Implications of the Higgs discovery for the MSSM”. doi:10.1140/epjc/s10052-013-2704-3

6. G. G. Ross, "SUSY: Quo Vadis?”. doi:10.1140/epjc/ s10052-013-2699-9

7. R. Catena, L. Covi, “SUSY dark matter(s)”. doi:10.1140/ epjc/s10052-013-2703-4

8. H. P. Nilles, “The strings connection: MSSM-like models from strings”. doi:10.1140/epjc/s10052-013-2712-3

9. B. Bellazzini, C. Csáki, J. Serra, “Composite Higgses”. doi:10.1140/epjc/s10052-014-2766-X

Open Access This article is distributed under the terms of the Creative Commons Attribution License which permits any use, distribution, and reproduction in any medium, provided the original author(s) and the source are credited.

Funded by $\mathrm{SCOAP}^{3}$ / License Version CC BY 4.0. 